\title{
Who Defines Excellence? An Intercultural Study Of Perceptions Of "Excellence" And The Effects Of These Interpretations In Global Higher Education
}

Veronica Velo, (E-mail: v.velo@ coventry.ac.uk), Coventry University, United Kingdom

\begin{abstract}
This article questions the perception of excellence in three different and geographically distant locations: Buenos Aires (Argentina), Coventry (UK) and Shanghai (China) and inquires about the capacity of educational systems to generalize globally the appreciation of student and university staff work. The main issue on which this article focuses is whether what higher education institutions consider as "bad", "good" or "excellent" is culturally sensitive, and if so, how to agree on objectives and procedures that are universally applicable. Current environmental conditions in the higher education landscape are pushing towards the development of international links between universities under different forms, such as franchised degrees, joint programs, mutual faculty and student exchanges, etc. As this new reality demands the homogenization of the curricula delivered as well as a compatible process of evaluation across borders, how could we achieve global excellence if what we understand by this term is culturally sensitive?
\end{abstract}

Key Words: excellence, education, curriculum, internationalization, repertory grids, culture.

\section{INTRODUCTION}

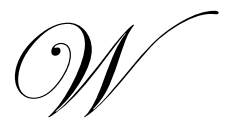

ho defines "excellence"? This notion, although objective in appearance, hides more than one meaning and leaves room for several interpretations to be constructed by individuals under the subconscious influence of their cultural environment. If interpretations of the term "excellence" are subjective, then it is possible to explain why we design performance measurement tools in education that might not be universally applied with the same level of effectiveness everywhere. In other words, when abstract concepts (like "excellence", for instance), are institutionally formulated as if they are concrete concepts, whereas their intrinsic subjectivity leaves room for different understandings of terms, this situation in general leads to intercultural confusion, frustration and mismanagement.

This article illustrates, through the example of the term "excellence" and its different interpretations in three different cities located in three different countries on three different continents, how sometimes higher education policy makers intend to quantify or qualify different ideas, characteristics, actions or results as if these were perceived in the same way all over the world whereas they may not be. In other words, if academics do not understand the same concept for the term "excellence" in the UK, in China and in South America, then how can levels of educational "excellence" in all these countries or in any others be compared when operating globally?

For example, if "excellence" in place A is intersubjectively linked to notions such as "punctual, wellgroomed, polite and reliable", whereas "excellence" in place B is intersubjectively linked to notions such as "inventive, creative, analytical and unconventional", then how could a unique evaluation system measure "excellence" in both places without unfairly favoring the activities taking place in one place rather than the other? If 
this was the case, it would not be surprising to notice that staff in the less favored location might feel diminished and therefore become demotivated and start to develop a bad attitude. Or, what might be even worse, staff might be inundated by a bureaucracy of manuals and contracts trying to put boundaries to the interpretations of the idea to be conveyed.

When overlooking the effects of intersubjective subconscious interpretations of notions, educators operating globally might be misleading our international partners at setting up and communicating objectives. Moreover, if curriculum planners do not understand the same for "excellence" universally, how can we create universal criteria for planning and controlling higher education international projects (research, joint degrees, etc.) of any kind?

Specifically, this article explores the values that are culturally linked to the notion of excellence in higher education at three different locations in three different continents: Buenos Aires (Argentina), Coventry (UK) and Shanghai (China). Using the Repertory Grids tool (methodology developed by psychologist George Kelly in 1955 and based on Western American values, and derived from the constructivistic school of thought), this article explores perceptions and intersubjective collective mental programmings related to the term "excellence" in the three cities that serve as basis for the study. The analysis of these mental charts will provide us with elements to determine in which ways different interpretations of the same notion ("excellence", in this case) might cause distortion in the implementation of performance appraisal systems or educational evaluation criteria in trasnational operations.

\section{LITERATURE REVIEW}

The Repertory Grids method (Kelly, 1955) has been widely used in clinical psychology (Winter, 1992), as it was developed to identify how agents create meaning. The basic idea is that humans understand reality through aligning concepts in their minds in order to make sense of the world. We comprehend life through comparisons of pairs of concepts by opposition to a third one. The criteria of comparison of these concepts are called "constructs" (Birch et al., 2001) and we link these constructs between them in order to organize our perception of reality (Smith, 2000). For example, we understand the concept of "cold" by contrasting terms "ice" - "winter" and "boiling kettle". Ice and winter share one characteristic that a boiling kettle does not. This allows us to build the construct cold/hot. Equally, we tend to link constructs, for example, we could establish a relationship between the constructs cold/hot and agreeable/disagreeable.

It is in the domain of psychology that this tool has been most widely used. In particular, it has proven its utility to deal with patients who cannot overcome depression because they relate the state of sadness to being superficial as opposed to being deep (Neimeyer, 1993). Nevertheless, applications of this method have been made in business (Pavlica \& Thorpe, 1998), as well as in other areas such as leisure studies (Botterill \& Crompton, 1996) and health care (Large \& Strong, 1985).

Fournier (1996) used Repertory Grids in the Human Resources field to analyze change in self-construction when young graduates enter the work market. She presented the case of two students having gone from university to employment and produced cognitive maps based on how these two people saw themselves in relation to other people before and after starting their professional lives.

Hunter and Beck (2000) used a laddering version of the Repertory Grids to explore similarities and differences in the way in which "excellent" systems analysts are viewed in Canada and in Singapore. They presented with a discussion on the validity of the research method in question and its applicability for qualitative research in general and cross-cultural studies in particular.

The scientific weight of the Repertory Grids method has been tested and re-developed by authors from diverse disciplines. Reeve, Owens and Neimeyer (2001) experimented with the use of different examples of constructs provided to participants in the framework of a particular experiment in psychology. The authors concluded that when they gave examples that were descriptive (constructs that would referred to a subjective or 
abstract personal characteristic such as generous/mean, reliable/unreliable, etc.), these pairs would elicit more personally revealing outcomes than when they were providing with examples of constructs that referred to factual dimensions (visible-concrete characteristics such as tall/short, young/older, national/foreigner, etc.). Smith (2000) examined statistically the reliability and validity of measures derived from repertory grids within 20 teachers from different primary schools in the UK who were asked to rate randomly chosen pupils of theirs over one year interval. The results regarding the constructs the teachers used to classify the students in the class one year later were statistically significantly convergent to the ones that they had used for a similar population one year before.

In this paper the Repertory Grids method will be used in order to point out which constructs are being produced by respondents from three different cultures and in which ways they link them up to other constructs.

\section{METHODOLOGY}

A modified version of the Repertory Grids (BBC Online, 2002) was used to test the constructs subconsciously developed by respondents from similar populations in Buenos Aires $(n=19)$, Coventry $(n=14)$ and Shanghai $(\mathrm{n}=20)$. All respondents were actively involved in the education system at the moment in which the survey took place, either as students, teachers or administrators. All respondents were from the same institution in their country.

Participants were requested to provide with constructs able to describe the differences they found between individuals of their choice that they considered excellent in a domain (excellent teacher, excellent actor, etc.) and others they would describe as average, using a modified version of the Repertory Grids test (see annex 1 for the document distributed). The instructions given to respondents on how to fill the document in were the following:

1. On the first row, each participant to the survey has to replace the descriptions provided by names of people they know that match that description. For example, replace "excellent teacher I" by "Maria" (because Maria is an excellent teacher), replace "excellent teacher II" by "Paul" (because Paul is also an excellent teacher), and replace "average teacher by "Sophie", because Sophie is an average teacher, and so on for all 15 characteristics.

2. Then, for each group of three people it is necessary to find a characteristic that two of these have, but one doesn't. For example, if Maria and Paul are outspoken and Sophie is introvert, then on the last column where it says "constructs", it has to be written "outspoken vs introvert" on the first line.

3. Repeat the same for each group of three people (and put the different characteristics that two of those have and the third doesn't have on the column on the right, one below the other).

4. Now go to each row (rows 1 to 5) and for each person you selected, put a cross if this person complies with one characteristic, and an "o" if the person doesn't. So, in our precedent example, for each one of the 15 people that you chose, you have to put an $\mathrm{x}$ on row 1 if the person is outspoken and a o if the person is introvert.

5. Same procedure for each pair of characteristics, on each row from 1 to 5 , put an $\mathrm{x}$ if the person complies with that characteristic, and an o if the person doesn't.

The application of that procedure allowed the identification of subconscious relationships established by participants between constructs while pointing out the pairs of concepts that carried highest links.

The constructs that allowed respondents to discriminate excellent individuals from average ones were then grouped in different categories: physical characteristics, personal characteristics, attitudes, and capacities. The total constructs belonging to each category was added up for each national sample and the number of relationships between the constructs of each category was added up in order to determine the mental mapping of concepts related to the notion of excellence in the three cultures. The results are presented in the next paragraphs. 


\section{DISCUSSIONS}

The groups of constructs chosen in each location are illustrated in the chart below.

Chart N. 1

Preferred groups of constructs by location

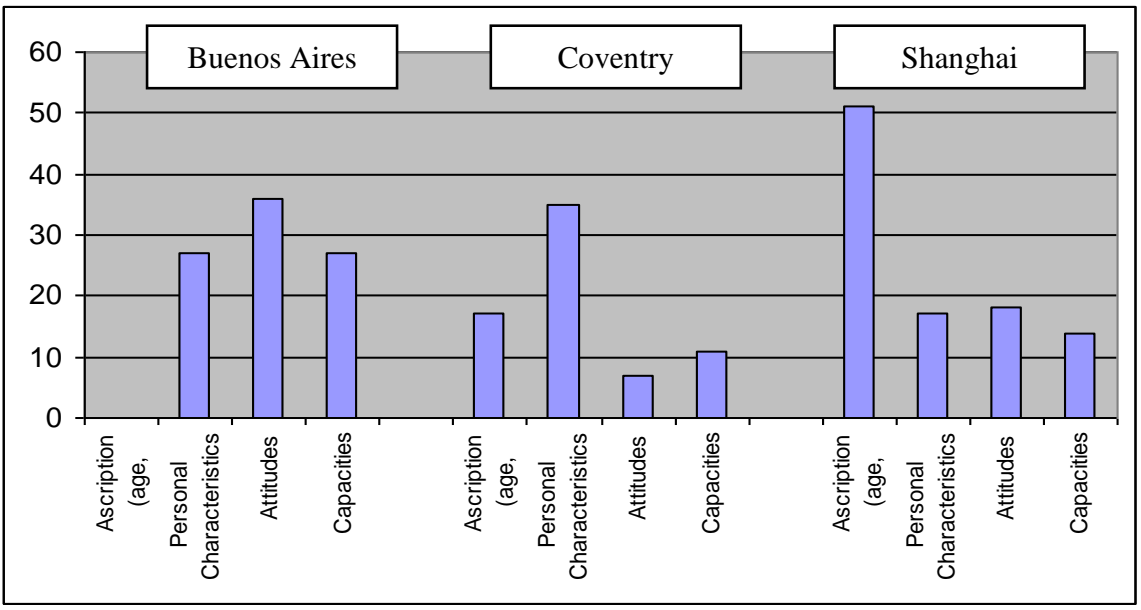

The first set of bars illustrates the groups of constructs chosen by the respondents from Buenos Aires, the second represents the answers obtained in Coventry and the third corresponds to the participants from Shanghai.

Cultural differences appear at first sight in this chart. Whereas in China, over $50 \%$ of respondents linked the notion of excellence to ascribed characteristics (age, social status, beauty, nationality, race), no Argentinean respondents did so. Respondents in England showed intermediary results, with a low percentage of constructs relating these concepts. In Buenos Aires higher education, the notion of excellence appears to be connected to the result of a balanced mix of personal characteristics (charm, warmth, and creativity), capacities (talent, know-how, skills) and the right attitude (hard work, persistence, honesty). Showing the right attitude appears to be in fact, the key determinant of excellence.

The results obtained in England show that personal characteristics are identified as the key determinant of excellence. Leadership skills in particular, as well as drive towards innovation were frequently mentioned. Ascribed qualities are considered important, but not as much as personality. Attitudes and capacities are not presented as paramount to achieve excellence. Charisma appears to be the concept that approaches the most the notion of excellence.

As per our sample from China, ascribed characteristics appear to be the most powerful determinant of excellence. Personal particularities, attitudes and capacities are put lower on the scale.

The above mentioned differences suggest the potential conflict that can appear when dealing internationally in education and the implications of this conflict. If, for example, a Chinese student travels to the UK hoping to be evaluated according to what he is, by opposition to by his/her leadership skills and capacity to innovate, then a high level of frustration is to be expected. Equally important, if an Argentinean student travels to England and expects to be assessed globally not only on his/her personal characteristics, but also on capacities and attitude, then disappointment, misperception and misunderstanding are to be faced.

Chart N.2 illustrates comparatively how each group links the notion of excellence to each group of constructs. 
Chart N. 2

Preferred groups of constructs

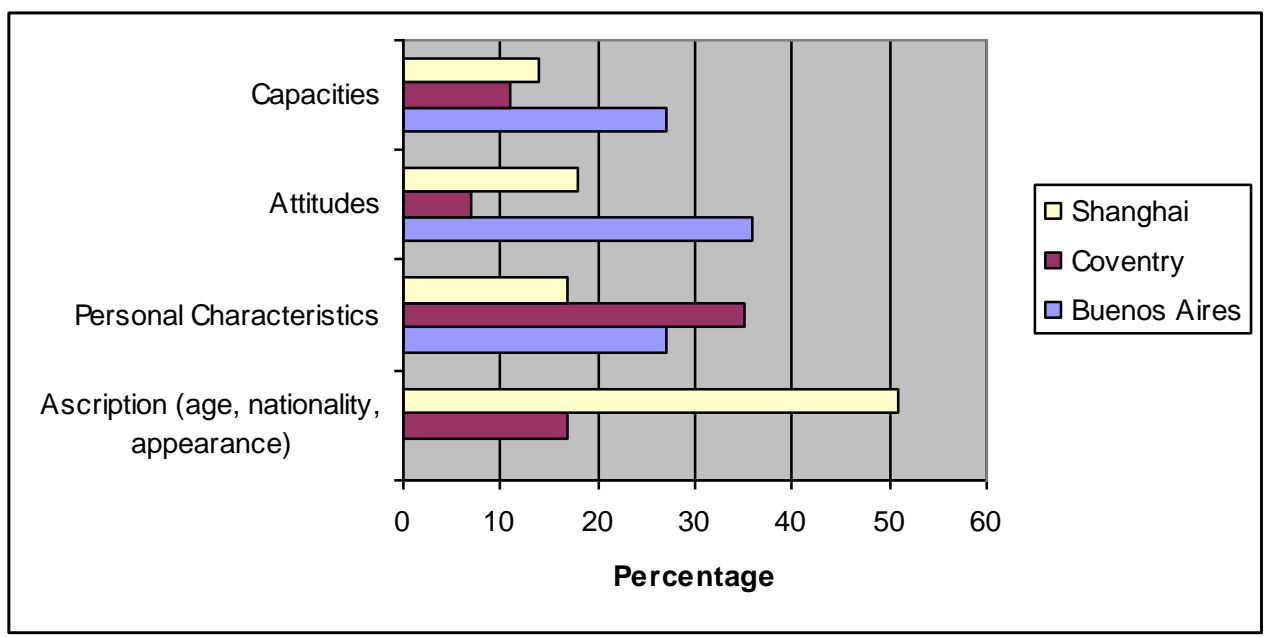

Having compared the preferred groups of constructs per location, the next step is to observe the links that the respondents from each culture made between groups of constructs.

The results obtained uncover once more the significant cultural differences and their implication in the conceptualization of the term "excellence" (see Chart N.3).

Chart N. 3

Preferred links between constructs for Buenos Aires

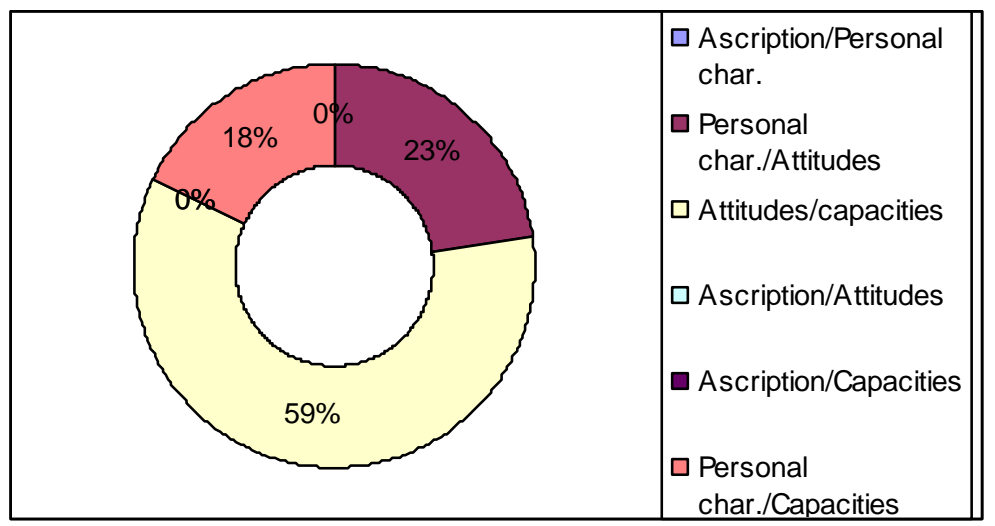

Results from the survey suggest that in Buenos Aires higher education, capacities and attitudes are very much considered as entwined. Someone who is capable of producing good work is expected to show a professional attitude. Or likewise, someone who shows a good attitude is perceived as someone who would produce good work. If we take into account the implications of these links, we will soon discover that in the evaluation systems of Argentinean Higher Education institutions, attitudes would tend to be compensated as much as capacity. "Trying hard" would be valued as high as actual results. A good student or an excellent student would certainly be described as the one who behaves correctly, has good intentions, and does not cheat. A bad student would be a student who shows a bad attitude. Also, vice-versa, a student that is weak at certain subjects could easily be penalized other than 
academically. Academic and behavioral punishment tend to mix. A student who has behavioral problems might probably lose points in his/her dissertation grade because his/her attitude is bad, so his/her capacity could be put under scrutiny.

An excellent student in the mind of the Argentinean respondents would be the one who is either smart (capable) or behaving according to the socially established rules (good attitude). This particular society would find it harder than others to differentiate between someone with good intentions to perform and someone who actually does perform.

Chart N. 4

Preferred links between constructs for Coventry

\begin{tabular}{|l|l|}
$\square$ Ascription/Personal \\
char. \\
$\begin{array}{l}\text { Personal } \\
\text { char./Attitudes } \\
\square \text { Attitudes/capacities } \\
\square \text { Ascription/Attitudes } \\
\square \text { Ascription/Capacities } \\
\square \text { Personal } \\
\text { char./Capacities }\end{array}$ \\
\hline
\end{tabular}

The respondents from the UK perceived ascription as a good predictor of personal characteristics. These results suggest the need for particular care to be taken not to stereotype when dealing with a diverse student population or with foreign partners or associates. Moreover, personal characteristics are also associated with attitudes. This means that certain behaviors could tend to be expected from people according to their affiliations. In an educational landscape that tends certainly towards globalization, immediate impressions on the expected outcomes from others and about their potential for excellence ought to be carefully kept under observation and scrutiny.

Chart N. 5

Preferred links between constructs for Shanghai

\begin{tabular}{|l|l|}
$\square$ Ascription/Personal \\
char. \\
$\square$ Personal \\
char./Attitudes \\
$\square$ Attitudes/capacities \\
$\square$ Ascription/Attitudes \\
$\square$ Ascription/Capacities \\
$\square$ Personal \\
char./Capacities
\end{tabular}


As shown in Chart N. 5, Chinese respondents said that personal characteristics (the way each individual is) are considered reliable predictors of what attitudes are to expect. This means that when dealing with the Shanghainese, it should not be surprising to discover that they will easily enquire about one's character and personality, as this is supposed to be an indicator of future behavior. Institutions being still quite behind in the development of a standardized system of legal procedures, trust becomes paramount. Therefore one would only deal with those they trust and one would only trust those who are perceived as having the right personality.

In the classroom, a Chinese student would generally tend to thoroughly scrutinize the teachers, and if possible to establish a personal connection in search of the true personality behind the role of the instructor. Once more, the notion of "guanxi" (Chinese term that emphasizes the importance of relationships and networks as a key aspect of society) becomes explanatory of most attitudes of this cultural group that would trust the person more than the rule.

\section{CONCLUSION}

The notion of excellence is certainly culturally sensitive. The values and associations that people from different countries make about this concept are consistently and significantly different.

In a time when the educational landscape calls for harmonization of curricula, standardization of procedures, and identical criteria for evaluation, forced uniformization would be costly. Training and even education in intercultural issues becomes an extenuating necessity for educational institutions that intend to operate globally. Otherwise, international students will interpret that they are being judged unfairly and local learners that have been raised through the values of their lecturers would be in a privileged position to succeed.

Values of a culture are not to be changed, but in the same way that a supplier has to adapt to the needs of his/her clients, international educational institutions must make as many efforts as possible to provide quality service, which includes understanding of the needs and backgrounds of their "consumers" if they seriously want to perform. Otherwise, international exchanges originally intended to increase cross-cultural tolerance and positive mutual enrichment will end up creating negative attitudes instead, and increasing stereotyping and misunderstanding between cultures.

\section{REFERENCES}

1. $\quad$ BBC Online (2002). All in the Mind (Online) available at www.bbc.co.uk/radio4/science/allinthemind_20021009.shtml. (Accessed on 23 November 2005).

2. Birch, M., Campbell, H., Danino, N., Gray, P., Gregory, P., Macfarlane, S., Nicholson, I. \& Read, J. (2001). Using Repertory Grids. Available at www.uclan.ac.uk/facs/destech/compute/research/conference/sept2001/nicholson-et-al.doc (accessed 23 November 2005).

3. Botterill, D. T. \& Crompton, J.L. (1996). Two case studies exploring the nature of the tourist's experience. Journal of Leisure Research, 28 (1), 57-82.

4. Fournier, V. (1996). Cognitive maps in the analysis of Personal Change During Work role Transition. British Journal of Management. 7: 87-105.

5. Hunter, G. \& Beck, J. (2000). Using Repertory Grids to Conduct Cross-Cultural Information Systems Research. Information Systems Research, 11 (1): 93-104.

6. $\quad$ Kelly, G. A. (1955). The Psychology of Personal Constructs. New York: Norton.

7. Large, R. \& Strong, J. (1985). The personal construct of coping with chronic low back pain: Is coping a necessary evil? Pain, 73(2), 245-252.

8. $\quad$ Neimeyer, R.A. (1994). The Threat Index and related methods. In R. A. Neimeyer (ed.), Death, anxiety handbook: Research, instrumentation and application. Washington, DC: Taylor \& Francis.

9. Pavlica K. \& Thorpe, R. (1998). Manager's perceptions of their identity: a comparative study between the Czech Republic and Britain. British Journal of Management, 9(2): 133-149. 
10. Reeve, J., Owens, G. \& Neimeyer, G. (2002). Using Examples in repertory Grids: The influence on construct Elicitation. Journal of constructivist Psychology, 15: 121-126.

11. Smith, H. (2000). The reliability and validity of structural measures derived from repertory grids. Journal of constructivist psychology. 13: 221-230.

12. Winter, D. A. (1992). Personal Construct psychology in clinical practice. London: Routledge.

\section{ANNEXES}

Annexe 1

Questionnaire distributed to participants in each location

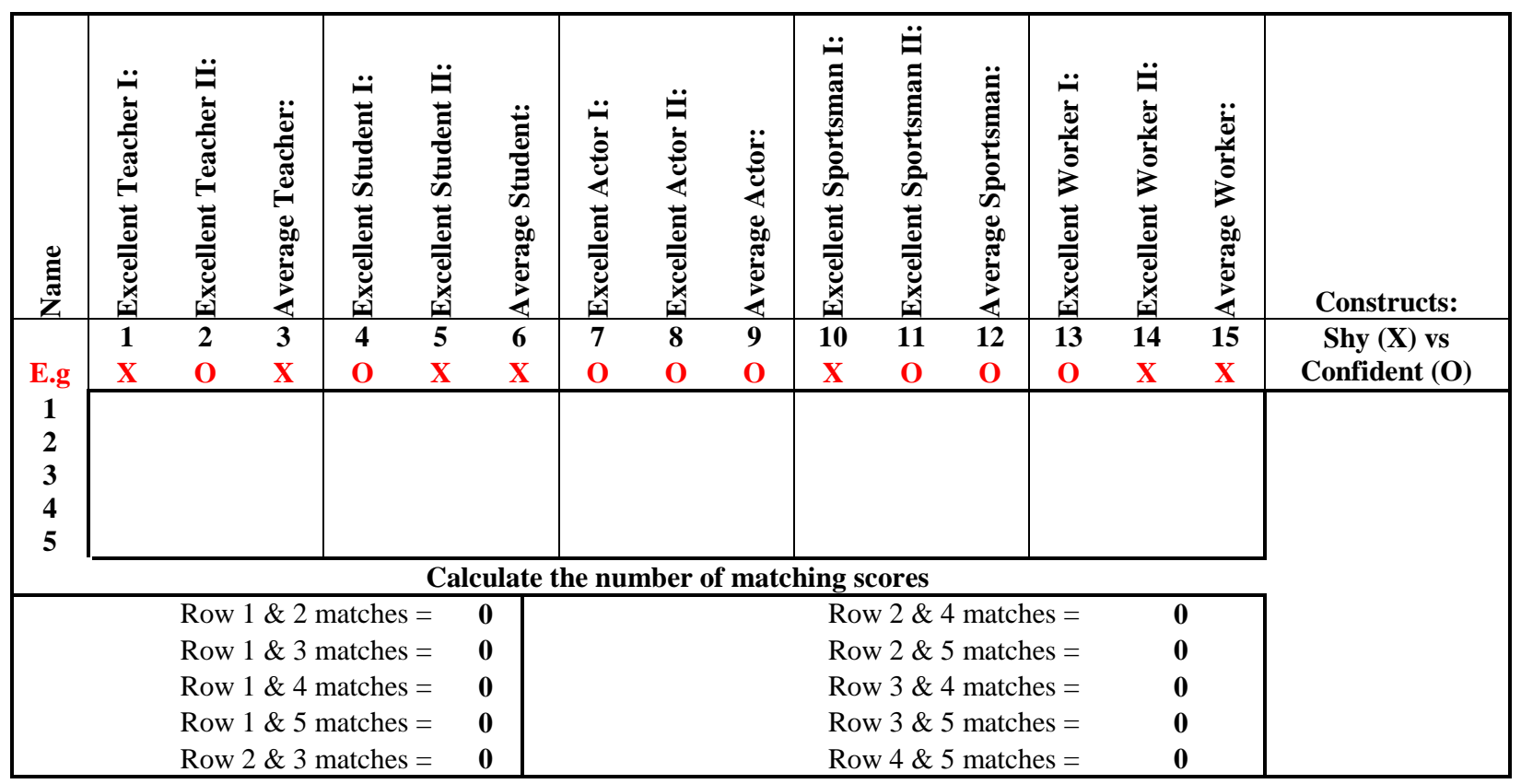

Source: www.bbc.co.uk/radio4/science/allinthemind 20021009.shtml 